\title{
A Preliminary Investigation into the Acquisition of Fingerprints on Food
}

Sarah Ferguson, Lynsey Nicholson, Kevin Farrugia, David Bremner, Dennis Gentles *

School of Contemporary Sciences, Forensic and Bio Sciences Division, University of Abertay, Dundee, UK

\section{* Corresponding Author:}

School of Contemporary Sciences

Division of Forensic and Bio Sciences

University of Abertay

Dundee DD1 1HG

United Kingdom

tel: +44 (0) 1382308110

fax: +44 (0) 1382308663

d.gentles@abertay.ac.uk 


\begin{abstract}
The potential for enhancement and recovery of latent fingerprints on a variety of foodstuffs has been investigated. In general, black magnetic powder and black powder suspensions appear to be the most successful enhancement techniques with a high number of ridge detail-developed prints over a selected time scale. Banana, apple and tomato surfaces showed enhancement of latent prints but potato and egg surfaces proved to be less successful.
\end{abstract}

Keywords: fingerprint, food, ageing, superglue, powder suspension 


\section{Introduction}

The surface onto which a fingerprint is deposited is often the primary decider as to which technique is selected for enhancement [1]. There is a vast range of such surfaces and specific enhancement techniques are selected based on the surface type and its porosity, the condition of the latent mark and the level of contamination which has occurred. Items of evidence that may retain fingerprints are often overlooked due to the belief that the item in question will not retain any fingerprints. This is mainly due to limited research on 'difficult surfaces' such as food, skin and fabric.

Singh et al. [2] determined that fingerprints could be successfully enhanced and recovered from food surfaces such as banana, apple and potato when using black powders although iodine fuming was also successful on apples. A further study by Trapecar and Vinkovic [3] focused on similar fruits and vegetables with some successful results. It was concluded that Swedish Black powder followed by special silver powder yielded the best quality of friction ridge detail and characteristics despite varying surface types. The process of cyanoacrylate fuming was also investigated [3] however results proved less successful. The food items were also graded in terms of their surface suitability with the tomato proving the most appropriate, followed by apple and banana, with the poorest results recorded on potatoes [3].

The surfaces of food items vary greatly not only in their texture and colouration, but also in their porosity and, like any item or surface undergoing development, each of these factors will undoubtedly affect the quality of visualisation achieved. The main aim of this study was to investigate a range of amelioration processes and ascertain which, if any, would be the most suitable for enhancing latent marks on specific food items. 


\section{Materials and Methods}

\section{Food Items}

Three fruits (apple, banana and tomato), three vegetables (onion, potato and pepper) and a dairy product (eggs) were selected as surfaces for testing. All substrates were collected in fresh form, stored in a refrigerator and used within a few days. Prior to fingerprint deposition, the food items were rinsed thoroughly with tap water and gently dried using clean, chemical free blue paper towel to ensure their surface was completely clean and free from any contaminants and unintended fingerprints. Finally the items were allowed to reach ambient conditions for about 24 hours. The food items were then marked off into five clearly labelled sections; one for each of the five fingerprint donors. Following fingerprint deposition, all food articles were stored at room temperature in normal lighting conditions awaiting enhancement at the allocated time intervals

\section{Fingerprint Deposition}

Donor suitability was checked by successful enhancement of fingerprints on a sheet of blank A4 paper with black magnetic powder. Five fingerprint donors were selected: 3 male (donors 1, 2, and 5) and 2 female (donors 3 and 4) who were instructed not to wash their hands at least an hour prior to the deposition of the print. Furthermore, the donors rubbed their fingers across their forehead and nose for about 10 seconds before rubbing their hands together and depositing 'loaded' fingerprints for a contact time of less than one second.

\section{Fingerprint Grading}

The following grading system (Table 1) was used to score the quality of ridge detail noted on developed fingerprints following each enhancement process.

\section{Enhancement Techniques}

The following development techniques were employed during the study: black magnetic powder, superglue fuming, ninhydrin, small particle reagent (SPR), black powder suspension (ready-made), white powder suspension (ready-made), and black powder suspension (freshly-made). To ensure that each chosen development method was working effectively a set of control fingerprints were deposited and enhanced 
daily on clear plastic surfaces along with each food items. Negative controls were performed for all techniques and substrates.

Table 1 - Grading of enhanced fingerprints

\begin{tabular}{|c|c|}
\hline Grade & Comments \\
\hline- & No visible fingerprint following enhancement. \\
\hline 1 & Visible print, however no ridge detail present. \\
\hline 2 & Visible print with slight discontinuous/dotty ridge detail. \\
\hline 3 & Visible print with approximately $1 / 3$ of ridge detail clear and continuous. \\
\hline 4 & Visible print with approximately $2 / 3$ of ridge detail clear and continuous. \\
\hline 5 & Visible print, whole mark present with clear and continuous ridge detail. \\
\hline
\end{tabular}

The substrates were examined using natural and artificial lighting at different angles. Any positive results obtained were recorded using a Nikon D50 digital SLR camera (set to manual focus) and equipped with a Nikon AF Micro-Nikkor 60mm f/2.8D lens. Following photography suitable prints were lifted with Remco ${ }^{\circledR}$ LP-200 latent print tape and labelled.

Ninhydrin, SPR and powder suspensions were prepared as recommended by the UK Centre for Applied Science and Technology (CAST) [4, 5]. Petroleum ether (40-60) was utilised instead of the expensive HFE-7100 for the preparation of ninhydrin.

\section{Black Magnetic Powder (BMP)}

This powder was purchased from CSI Equipment Ltd (UK), Batch 96528. [Black Mag]

\section{$\underline{\text { Superglue }}$}

Hard Evidence superglue (in liquid form), Product 95318, Batch 7553 was purchased from CSI Equipment before fuming in a bespoke superglue cabinet manufactured by Electronic Services, 20 Hopefield Avenue, London NW6 6LH. 


\section{Ninhydrin}

Concentrated Solution: ninhydrin (25g, Sigma) was dissolved in absolute ethanol (225mL, Sigma). Ethyl acetate $(10 \mathrm{~mL}$, Sigma) followed by acetic acid $(25 \mathrm{~mL}$, Sigma) was added to the slurry and stirred until a clear yellow solution was produced.

Ninhydrin Working Solution: ninhydrin concentrated solution $(52 \mathrm{~mL})$ was added with stirring to petroleum ether $(40-60 ; 948 \mathrm{~mL})$ to make $1 \mathrm{~L}$ of working solution.

Treatment of articles with Ninhydrin: The articles were immersed in the working solution for a maximum of five seconds and the excess solution was allowed to drain back in the tray. The item of food was allowed to dry completely before heating in a humidity oven at $80{ }^{\circ} \mathrm{C}$ for four minutes at a nominal $65 \%$ relative humidity. Enhancement occurred immediately or within the next few hours/days depending on the substrate.

\section{$\underline{\text { Small Particle Reagent (SPR) }}$}

Concentrated solution: Aerosol OT solution $(10 \% ; 7.5 \mathrm{~mL}$, Sigma) was added to tap water $(500 \mathrm{~mL})$ and stirred to create a detergent solution. Molybdenum disulphide powder (50g, Sigma) was added followed by a small amount of detergent solution until a smooth paste was produced. Half of the remaining detergent solution was added to create a slurry which was then transferred to a $1 \mathrm{~L}$ bottle and the remaining detergent solution was added.

Working solution: The concentrated solution was shaken vigorously and transferred to a $5 \mathrm{~L}$ bottle. Tap water (3L) was added to the concentrated solution.

Treatment of articles with SPR: The working solution was agitated prior to transferring a suitable volume for complete immersion of the food item into a basin. After a suitable time the item was removed from the suspension and rinsed with distilled water. 
Black powder suspension (Ready and freshly made solutions)

Wetwop $^{\mathrm{TM}}$ ready made solutions were purchased from WA Products, UK [Black PS (RM)]. A freshly made powder suspension was prepared by adding iron (II/III) oxide (Fisher, 20g) to $20 \mathrm{~mL}$ stock detergent solution with stirring until no lumps remained. The suspension was then applied to the articles to be examined using a soft animal hair brush [BPS (FM)].

Detergent solution: Photoflo $(500 \mathrm{~mL})$ was added to distilled water $(500 \mathrm{~mL})$ and stirred continuously for 10 minutes. The solution will keep indefinitely.

\section{Black powder suspension [New (20g) BPS and New (10g) BPS]}

Two formulations were investigated with different weights of iron oxide (II/III) and using distilled water rather than detergent stock solution. Iron oxide (II/III) (10g and $20 \mathrm{~g}$, Fisher) was added to distilled water $(20 \mathrm{~mL})$ and mixed, ensuring no lumps remained before applying to the sample with a soft animal hair brush. This change in formulation helped the powder suspension adhere better to the surfaces of the food items being treated. The solution has a very short life and was used immediately.

\section{White powder suspension (Freshly made solution)}

Titanium dioxide (10g, Sigma) was weighed and $20 \mathrm{~mL}$ of stock detergent solution was added with stirring until no lumps remained. The suspension was then applied using a soft animal hair brush. Occasionally titanium dioxide (20g) was used to 'thicken' the solution further. [White PS(FM)]

\section{Fingerprint Ageing}

Examples of each food type: apple, banana, tomato, potato, onion, pepper, and egg were prepared each week and developed over a two week period as follows: 2 hours, 1 day, 2 days, 3 days, 4 days, 7 days , and 14 days after deposition. This process was then repeated for all enhancement techniques under investigation. 


\section{Results and Discussion}

\section{Evaluation of Enhancement Techniques}

A negative control ensured that the treatments used did not react with the surfaces of the materials examined. Figure 1 shows the overall percentage of any positive enhancement (i.e. 1-5) achieved for each development process. Similar to previous studies [2, 3], black magnetic powder scored highly (51) for the enhancement of fingerprints on food while SPR and the black powder suspensions prepared with distilled water were a close second (49). In comparison, white powder suspension showed poor enhancement (4) on the food items in this study. No prints were obtained with superglue fuming but the technique did make it possible to observe where the mark had been deposited, however, the ridges were distorted due to lumps of superglue which had formed. In most cases, it was found that when a substrate was left for ten to fifteen minutes, after treatment with superglue, the detail in the print became clearer. No enhancement was observed with ninhydrin and there was no difference in the results obtained when it was applied via immersion or brush technique. This is perhaps due to the moisture-containing surfaces of the fruits and vegetables. Furthermore, the ninhydrin process caused the banana skins to turn black.

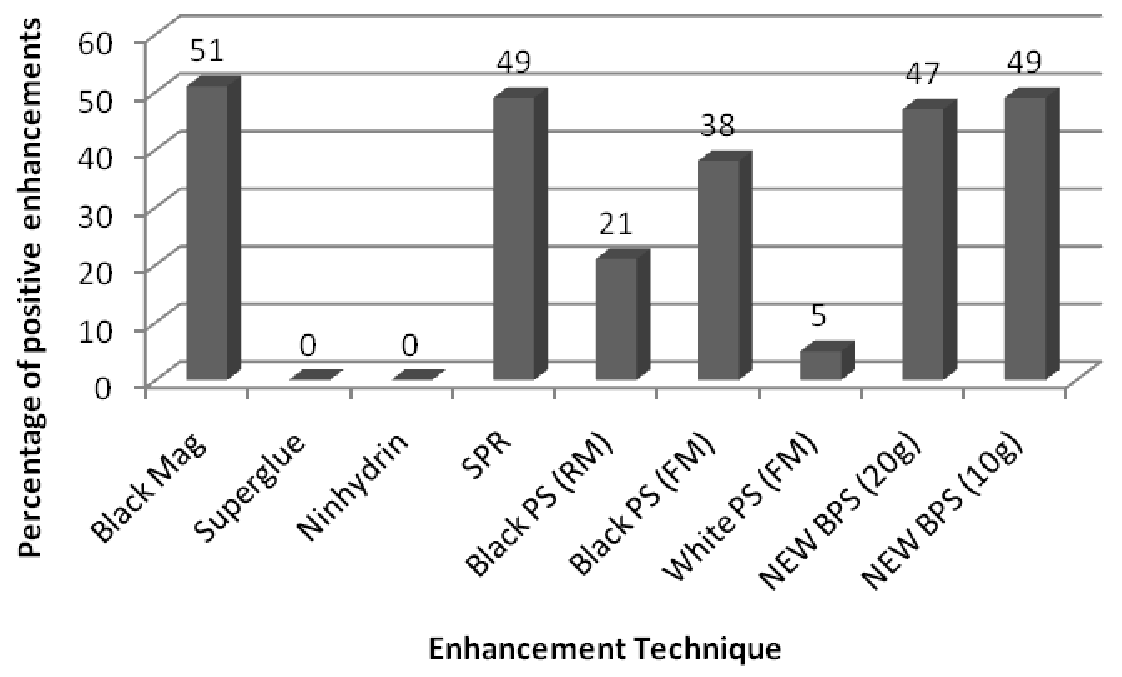


Figure 1 - Postive Grading of fingerprints by enhancement technique

Table 2 and figure 2 show the detailed gradings obtained for each technique where black magna powder appears to be the most successful at enhancing fingerprints on food producing with the highest overall number of positive enhancements at $51 \%$ and the highest number of enhanced marks with a quality rating of 5. Superglue fuming and ninhydrin have been omitted from the graph as no enhancement was achieved with these techniques.

Table 2 - Overall quality ratings achieved arranged by technique

\begin{tabular}{|c|c|c|c|c|c|}
\hline & Rating 1 & Rating 2 & Rating 3 & Rating 4 & Rating 5 \\
\hline $\begin{array}{c}\text { Black Magna } \\
\text { Powder }\end{array}$ & 37 & 31 & 20 & 22 & 14 \\
\hline $\begin{array}{c}\text { Superglue } \\
\text { fuming }\end{array}$ & 0 & 0 & 0 & 0 & 0 \\
\hline Ninhydrin & 0 & 0 & 0 & 0 & 0 \\
\hline SPR & 81 & 24 & 11 & 5 & 0 \\
\hline $\begin{array}{c}\text { Black PS } \\
\text { (Ready made) }\end{array}$ & 15 & 7 & 0 & 0 & 0 \\
\hline $\begin{array}{c}\text { Black PS } \\
\text { (Freshly } \\
\text { made) }\end{array}$ & 19 & 19 & 9 & 4 & 2 \\
\hline $\begin{array}{c}\text { White PS } \\
\text { (Freshly } \\
\text { made) }\end{array}$ & 3 & 4 & 4 & 1 & 0 \\
\hline $\begin{array}{c}\text { New (20g) } \\
\text { Black PS }\end{array}$ & 30 & 31 & 25 & 23 & 5 \\
\hline $\begin{array}{c}\text { New (10g) } \\
\text { Black PS }\end{array}$ & 2 & 35 & 35 & 21 & 6 \\
\hline
\end{tabular}




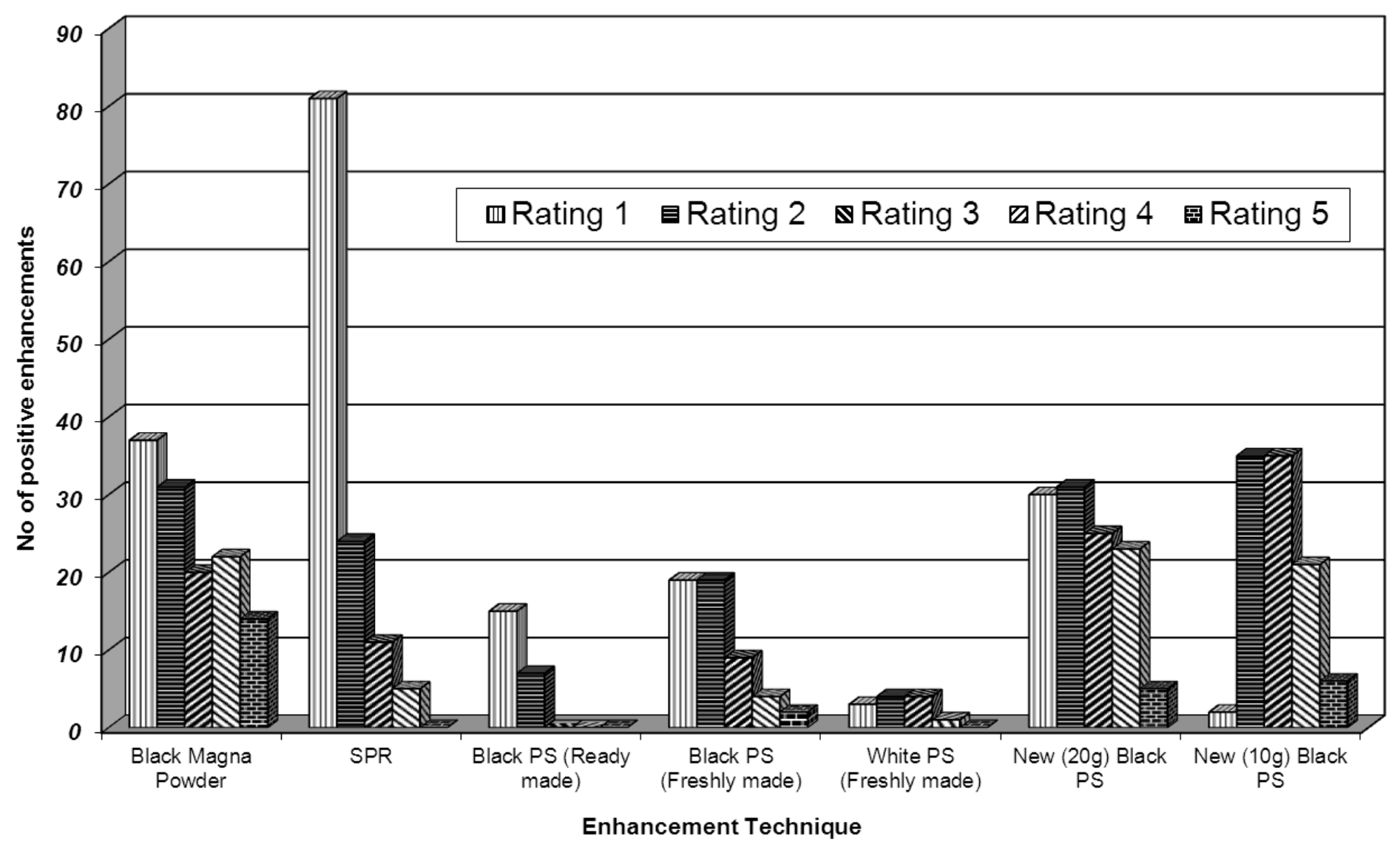

Figure 2 - Ratings (1-5) achieved by different techniques 
As the porosity of the food items was not known prior to testing it was expected that results for each enhancement process would vary on each surface type. Figure 3 demonstrates the number of positive enhancements achieved on each food item using each development processes.

With 34 visible marks, banana yielded the highest number of positive enhancements using the new formulation of black powder suspension [new (10g) BPS], with tomato showing the second highest value (28 marks) and onion next (26). Interestingly the new (10g) BPS showed no enhancement of latent prints for potato and very little enhancement for egg. Banana also showed the best result for the new (20g) BPS (31), with tomato next (22). However, the new (20g) BPS proved to be the most effective reagent overall for apple (21), although the new (10g) BPS was a close second (14). The best results for potato (14) and egg (11) were achieved using the SPR though generally it was not that effective and the SPR gave superior results for onion (31), banana (28) and pepper (17). The black magnetic powder proved to be the most successful at enhancing marks on both onion (32 positive results) and pepper (28) and banana (28).

In general, banana and onion yielded the highest number of positive gradings and the least successful surfaces for development were potato and egg which both gave the lowest number of positive enhancments for most techniques, or no positive results at all. If the number of positive marks are totalled for all food items, BMP (121) is marginally better than new (10g) BPS (119) and SPR (118). Although lifting of developed marks was investigated, no benefit was observed from lifts. Most surfaces were quite difficult to lift from due to the shape of the substrates but the most successful fingerprint lifting was from bananas, onions and tomatoes possibly due to their very smooth surfaces. 


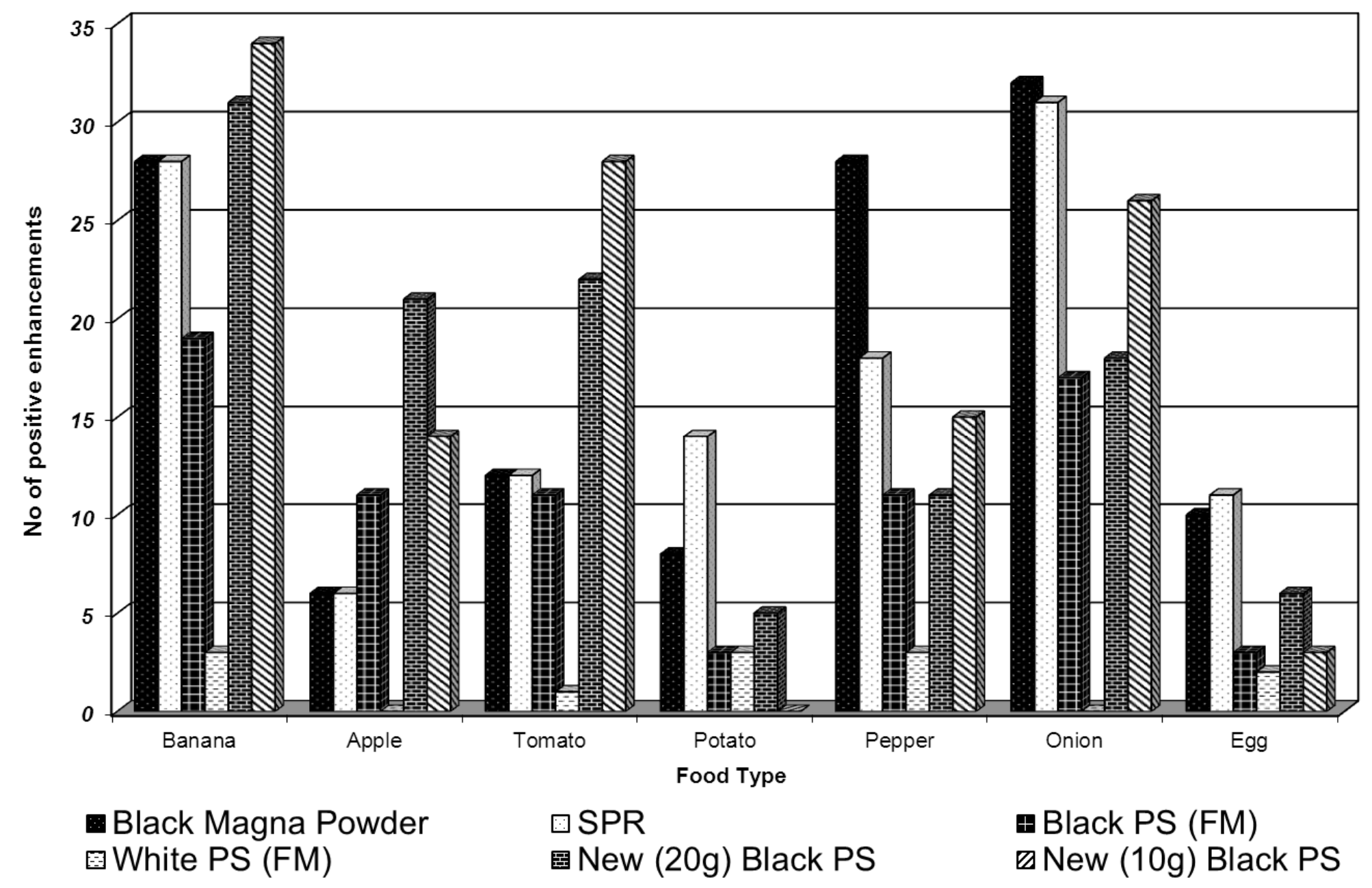

Figure 3 - Overall positive gradings on each food surface 


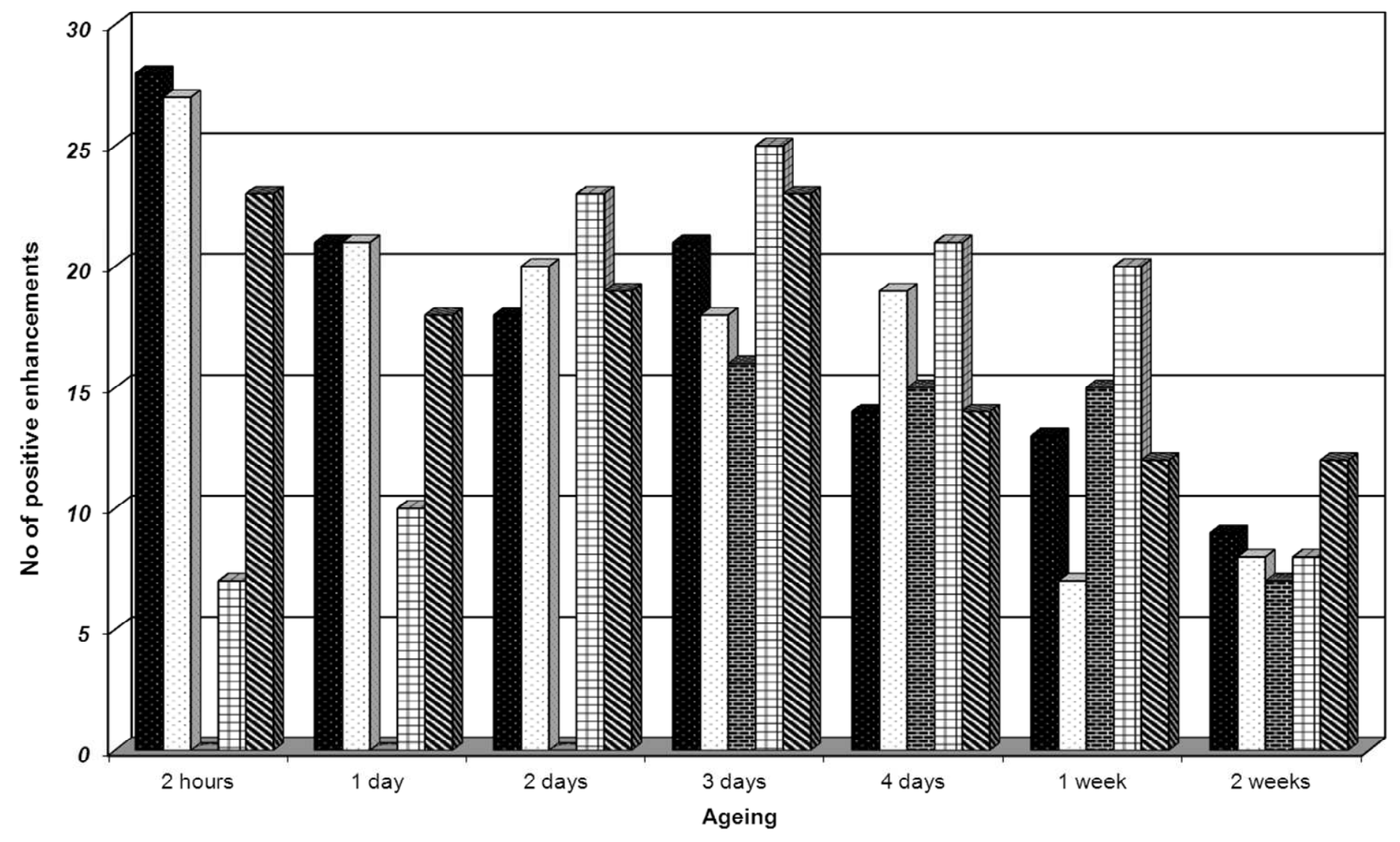

Black Magna Powder $\square$ SPR Black PS (FM) $⿴$ New (20g) BPS New (10g) BPS

Figure 4 - Comparison of enhancement technique effectiveness over time 
Figure 4 compares the number of positive gradings for each technique over time of fingerprint deposition and subsequent enhancement. Superglue fuming, ninhydrin, White PS (FM) and Black PS (RM) have been omitted from the graph to aid clarity as these enhancement techniques were shown to be inferior to the others as seen in figure 1. Generally, there is a decrease in the number of positive enhancements as the time since deposition increases. In particular, Black Magna Powder, SPR and new (10g) BPS showed a decrease over the 2 weeks. The value is 0 for Black PS (FM) for 2 hours, 1 day and 2 days because no experiments were performed and not because of negative results and again there is a general trend downwards for the results obtained. Interestingly, the new (20g) BPS showed an increase in the number of positive enhancements from 2 hours up to 3 days before falling again from 3 days onwards. This may be due to the mark drying out and further research would be required to verify this. It is also worth noting that the BPS (RM)is based on carbon black whilst the BPS (FM) is based on iron oxide powder which may account for the differences in performance.

\section{Examples of fingermarks observed}

Figures 5-9 show photographs of some of the enhanced fingerprints on food achieve by the various techniques.

\section{Evaluation of Powder Suspensions}

Figure 10 illustrates the overall number of positive gradings achieved using different formulations of powder suspension at different time intervals. The ready made black powder suspension [Black PS (RM)] and White PS (FM) only provided a small number of positive gradings. The Black PS (FM) yielded a higher number of positive gradings for prints aged 3 days and more. However, the highest number of positive gradings was obtained with the use of the new formulations of powder suspensions. Both new (10g) BPS and new (20g) BPS showed the best enhancements with the former being best suited for substrates that were prone to high background staining such as the onion. 


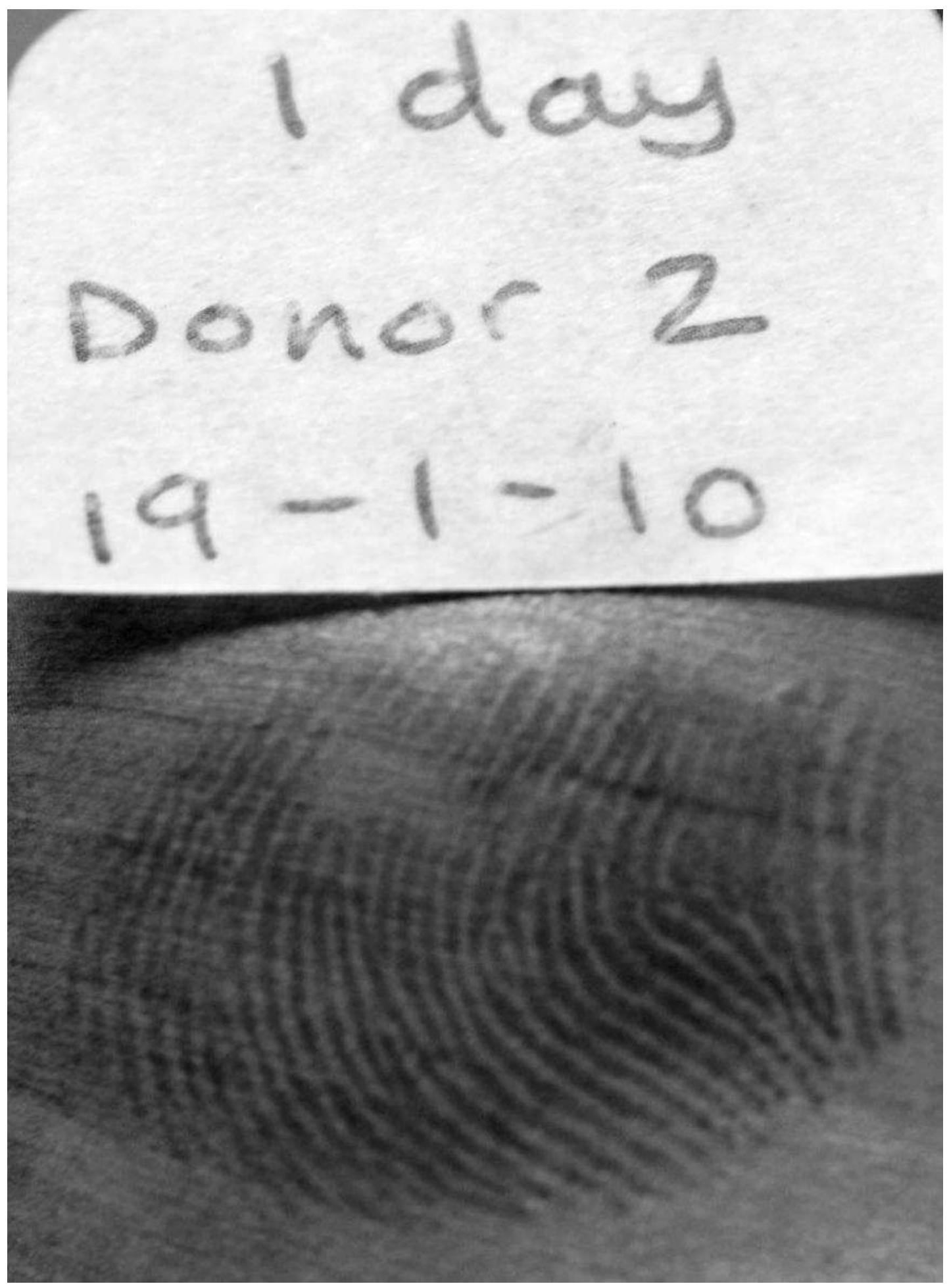

Figure 5 - Fingerprint enhancement with black magnetic powder for 1 day old depositions on an onion.

16 


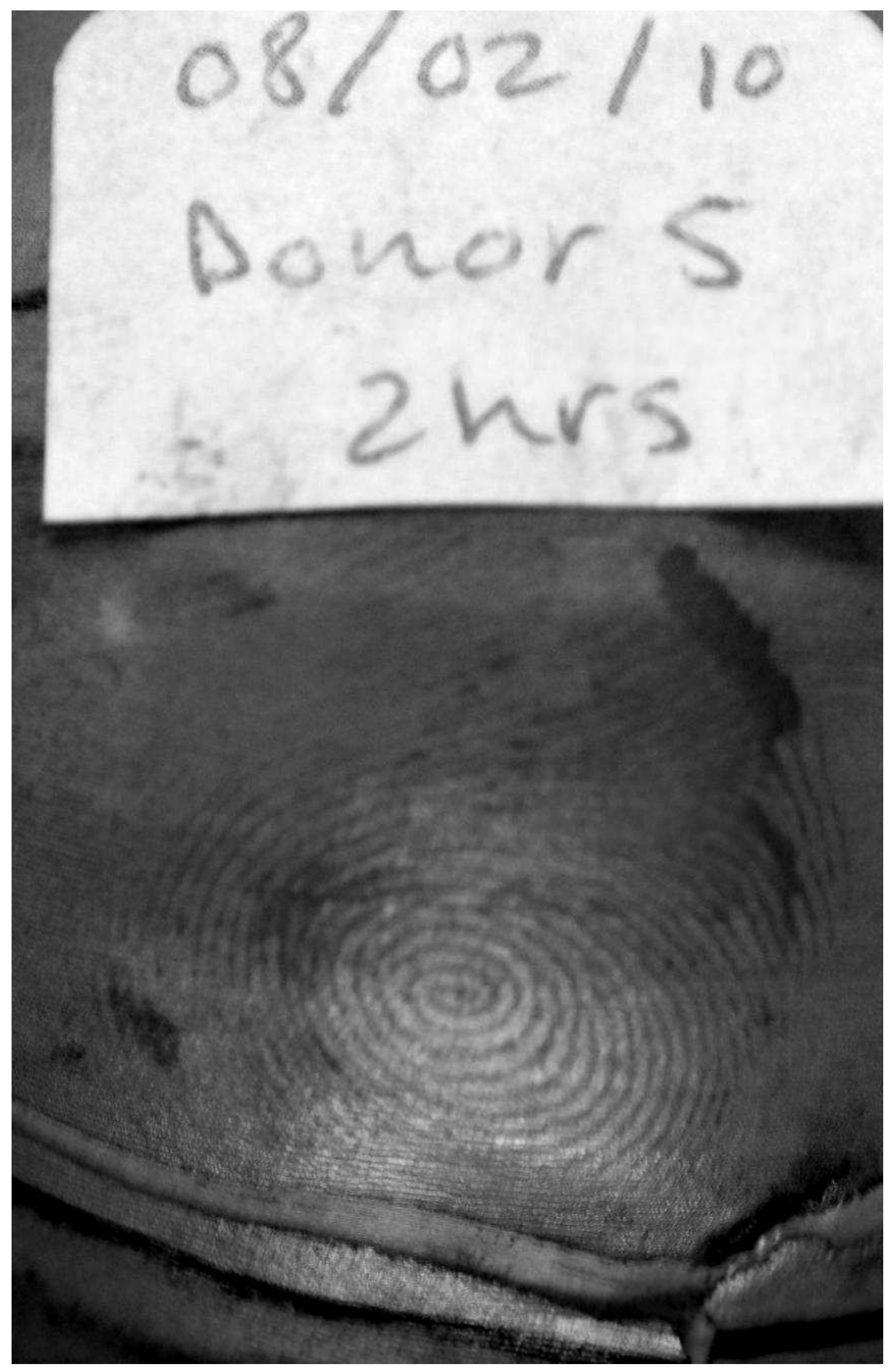

Figure 6 - Fingerprint enhancement with SPR for 2hour deposition on onion 


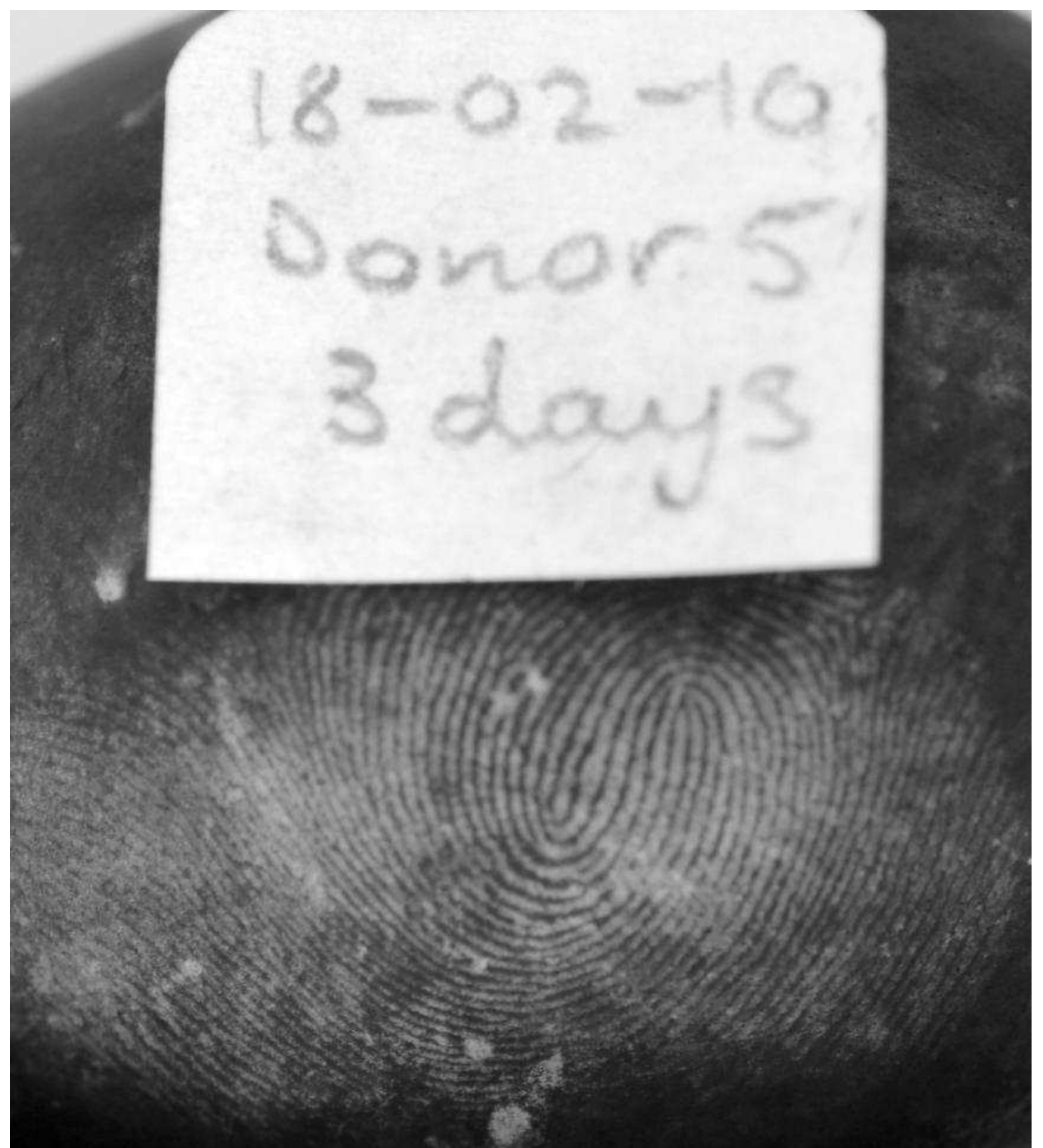

Figure 7 - Fingerprint enhancement with freshly made black powder suspension for 3 day deposition on an egg 


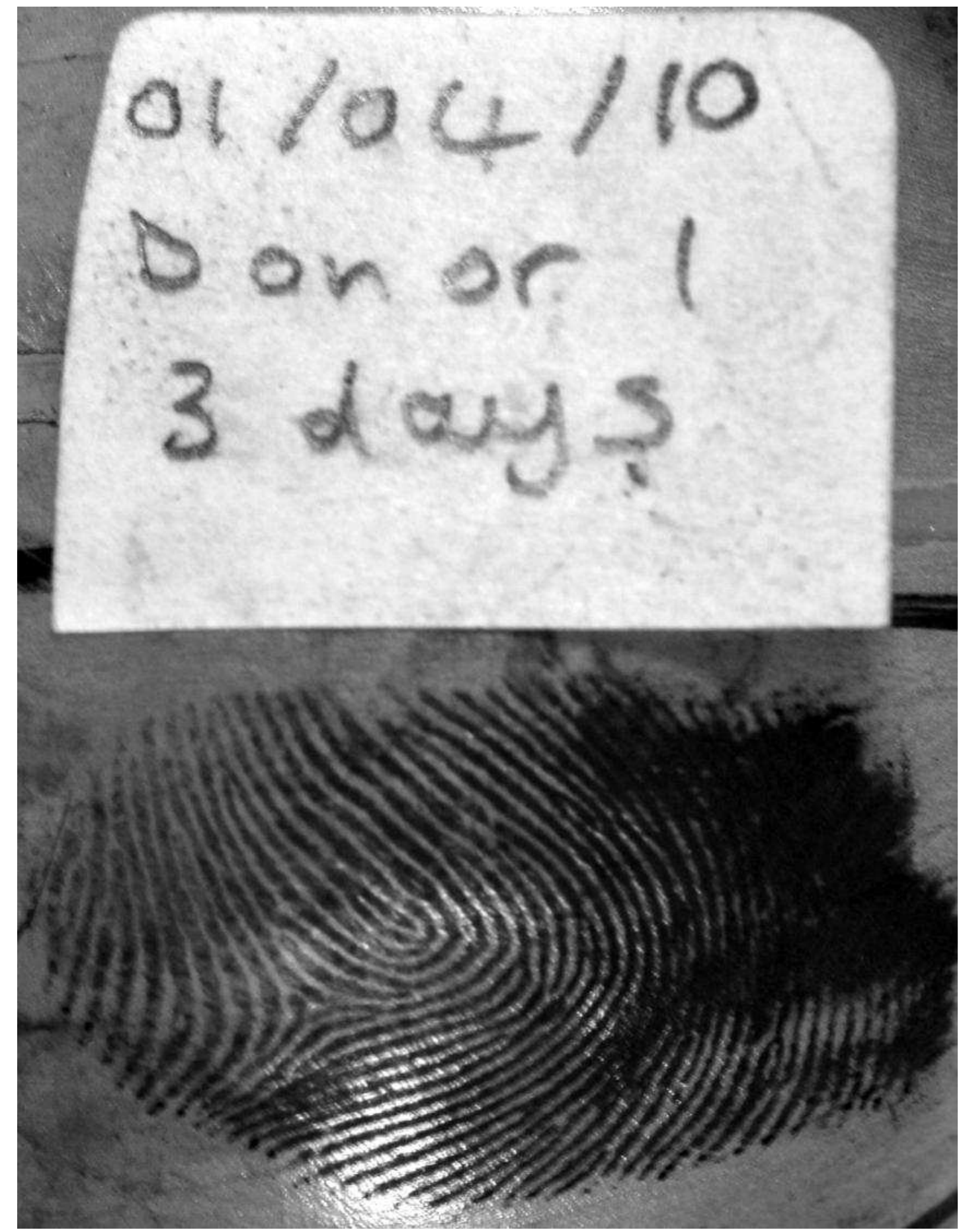

Figure 8 - Fingerprint enhancement with freshly made new formulation of black powder suspension $(20 \mathrm{~g})$ for 3 day deposition on onion. 


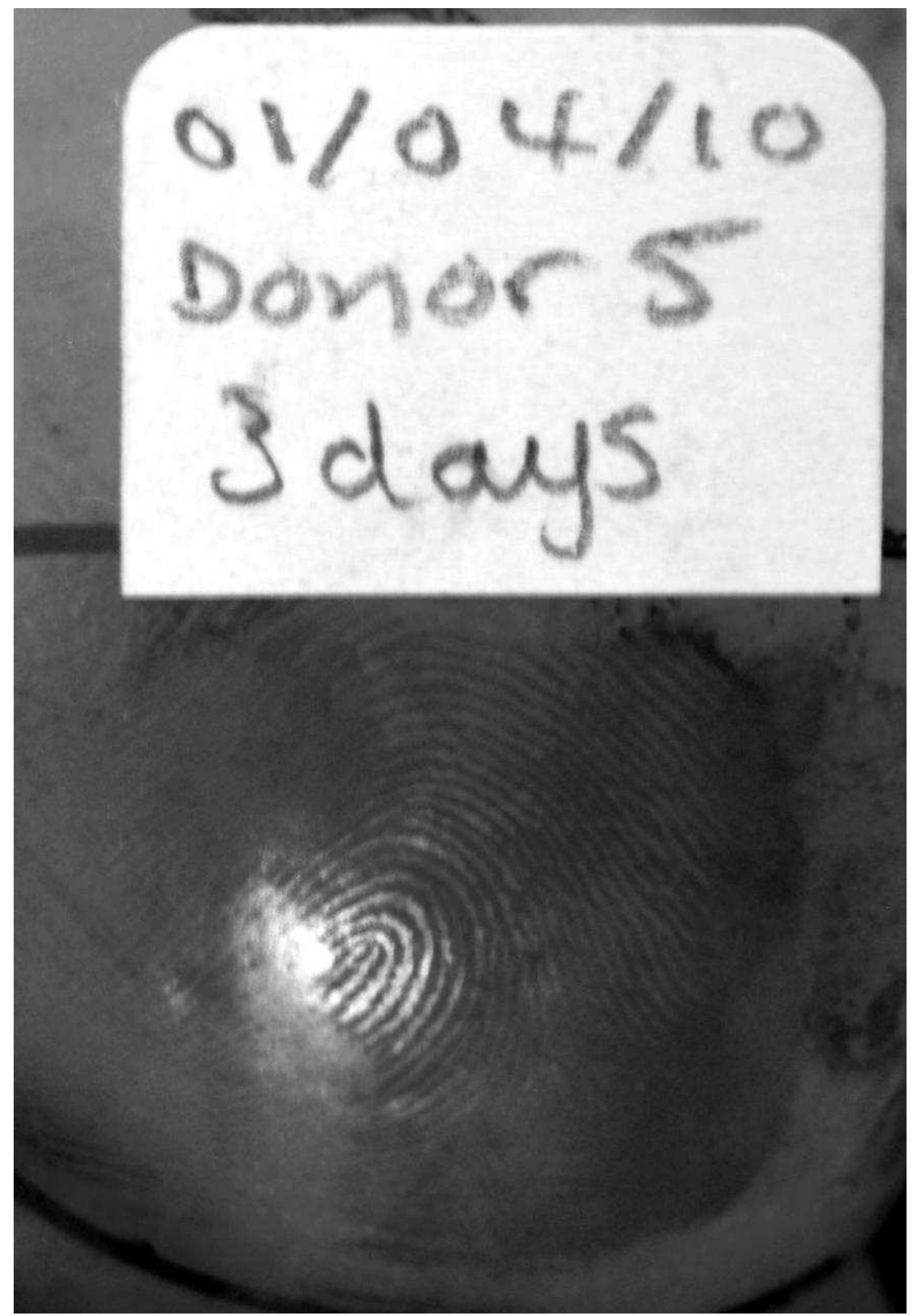

Figure 9 - Fingerprint enhancement with new formulation of black powder suspension $(\mathbf{1 0 g})$ for 3 day deposition on tomato 


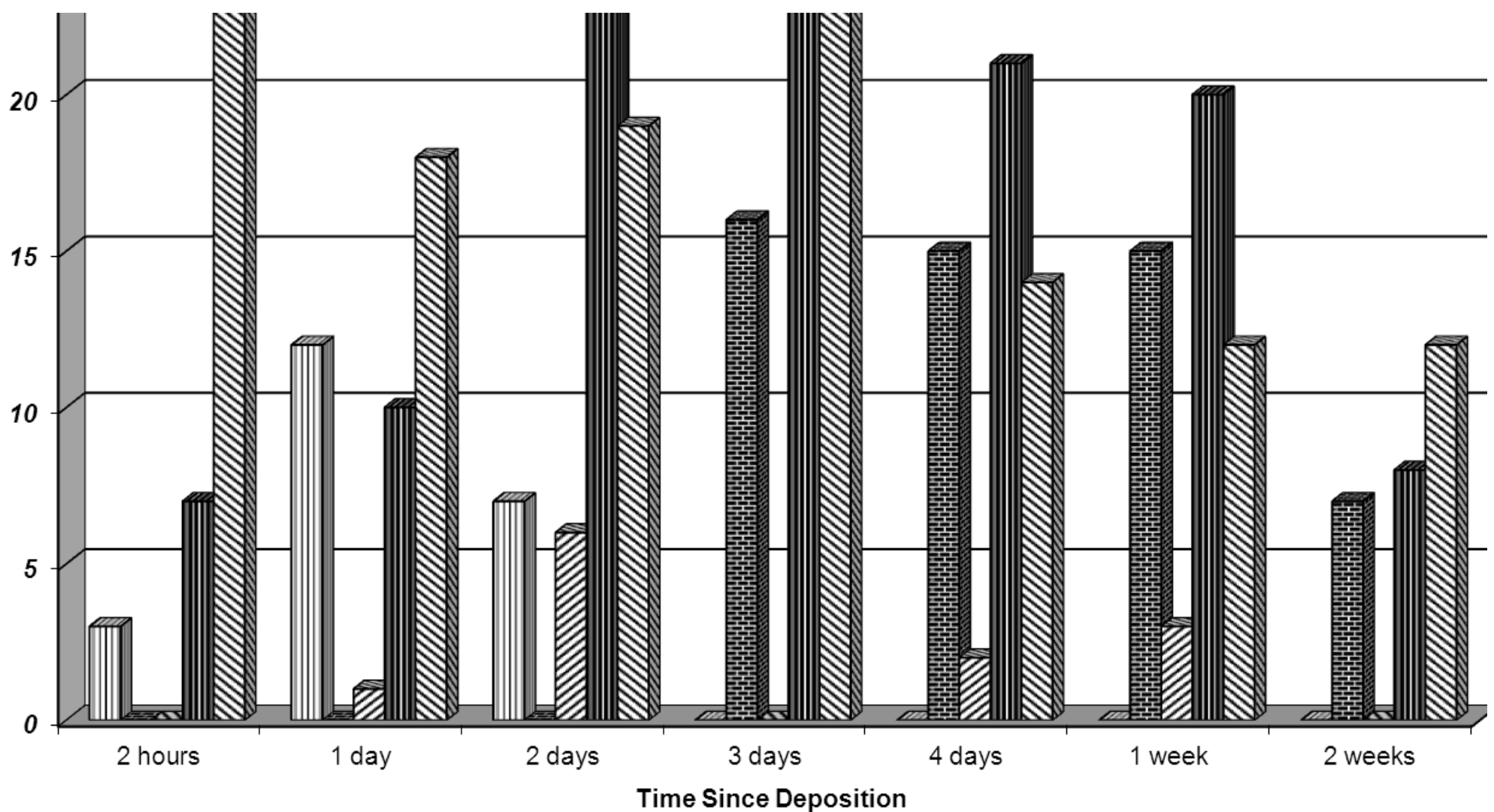

ack PS (RM) 国Black PS (FM) 『White PS (FM) 血 New (20g) Black PS 『New (10g) Black

Figure 10 - Comparison of powder suspensions and time intervals. 


\section{Conclusions}

Only two papers have previously been published in relation to the acquisition of fingerprints on foodstuffs $[2,3]$. Both papers concluded that using black powder is the most suitable development technique with the later paper [3] specifying Swedish Black Powder. Our study has shown that Black Magnetic powder developed more highly rated fingerprints than other techniques but that Small Particle Reagent developed more than twice the amount of fingerprints albeit at a lower rating. Our study also included the use of Powder Suspensions (PS) and, whilst the commercially available PS solutions developed less marks than Black Magnetic Powder, adaptations to this technique proved to develop almost as many marks as the powder and SPR with many more marks rated in the mid quality than the other techniques. As a result of work described herein it has been shown that by altering the Powder Suspension formulation so that it is more dilute than that recommended by the Home Office Manual of Fingerprint Development Techniques, the technique is more effective than BMP on certain food stuffs. The use of the $10 \mathrm{~g}$ and $20 \mathrm{~g}$ BPS, rather than the recommended or indeed the readymade powder suspensions that are commercially available, yielded some extremely promising results and with further research and development of powder suspensions it may be possible to develop a formulation that may produce developed marks of high quality that will outperform the BMP method of enhancement.

Furthermore, this study demonstrates that the enhancement and recovery of latent marks on food items is possible. Black magnetic powder proved to be the most successful, achieving an average of over $50 \%$ positive enhancements. Banana and onion appear to be the best suited surfaces in this study for the lifting of latent fingerprints whereas potato and egg were the least successful surface. As fingerprints age there are a variety of results though generally newer prints give more positive enhancements. Powder suspensions provided promising results and also appeared to provide better results with ageing of the fingerprint. Future work will include statistical analyses of data as well as an assessment of recent formulations of powder suspensions recommended by the UK Home Office Centre for Applied Science and Technology where the detergent solution is prepared from Triton X-100 and ethylene glycol. 


\section{References}

1. Morimoto, S.; Kaminogoa, A.; Hiranob, T. A New Method to Enhance Visualization of Latent Fingermarks by Sublimating Dyes, and its Practical Use with a Combination of Cyanoacrylate Fuming. Forensic Science International 1998, 97 (2), 101-108.

2. Singh, G.; Sodhi, G.S.; Jasuja, O.P. Detection of latent fingerprints on fruits and vegetables. Journal of Forensic Identification 2006, 56 (3), 374-381.

3. Trapecar, M.; Vinkovic, M.K. Techniques for fingerprint recovery on vegetable and fruit surfaces used in Slovenia -- A preliminary study. Science \& Justice 2008, 48 (4), 192-195.

4. Bowman, V. 2005. Manual of Fingerprint Development Techniques. 2nd ed. Sandridge, UK: Home Office Scientific Development Branch.

5. Home Office Scientific Development Branch. Additional Fingerprint Development Techniques for Adhesive Tapes. HOSDB Publication March 2006, 23/06. 